\title{
Periodic Solutions of Duffing Equation with an Asymmetric Nonlinearity and a Deviating Argument
}

\author{
Zaihong Wang, Jin Li, and Tiantian Ma \\ School of Mathematical Sciences, Capital Normal University, Beijing 100048, China \\ Correspondence should be addressed to Zaihong Wang; zhwang@cnu.edu.cn
}

Received 24 July 2013; Accepted 28 October 2013

Academic Editor: Irena Rachůnková

Copyright (C) 2013 Zaihong Wang et al. This is an open access article distributed under the Creative Commons Attribution License, which permits unrestricted use, distribution, and reproduction in any medium, provided the original work is properly cited.

\begin{abstract}
We study the existence of periodic solutions of the second-order differential equation $x^{\prime \prime}+a x^{+}-b x^{-}+g(x(t-\tau))=p(t)$, where $a, b$ are two constants satisfying $1 / \sqrt{a}+1 / \sqrt{b}=2 / n, n \in N, \tau$ is a constant satisfying $0 \leq \tau<2 \pi, g, p: R \rightarrow R$ are continuous, and $p$ is $2 \pi$-periodic. When the limits $\lim _{x \rightarrow \pm \infty} g(x)=g( \pm \infty)$ exist and are finite, we give some sufficient conditions for the existence of $2 \pi$-periodic solutions of the given equation.
\end{abstract}

\section{Introduction}

In this paper, we are concerned with the existence of periodic solutions of the second-order differential equation with an asymmetric nonlinearity and a deviating argument:

$$
x^{\prime \prime}+a x^{+}-b x^{-}+g(x(t-\tau))=p(t),
$$

where $a, b$ are two constants satisfying $1 / \sqrt{a}+1 / \sqrt{b}=2 / n$, $n \in N, \tau$ is a constant satisfying $0 \leq \tau<2 \pi, g, p: R \rightarrow R$ are continuous, and $p$ is $2 \pi$-periodic.

In recent years, the periodic problem of the second-order differential equation with a deviating argument has been widely studied because of its background in applied sciences (see [1-6] and the references cited therein).

In case when $\tau=0$ and $a=b=n^{2}$, (1) becomes

$$
x^{\prime \prime}+n^{2} x+g(x)=p(t) .
$$

Assume that the limits

$$
\lim _{x \rightarrow \pm \infty} g(x)=g( \pm \infty)
$$

exist and are finite. Lazer and Leach [7] proved that (2) has one $2 \pi$-periodic solution provided that the function

$$
\Psi(\theta)=2[g(+\infty)-g(-\infty)]-\int_{0}^{2 \pi} p(t) \sin n(t+\theta) d t
$$

is of constant sign.
In case when $\tau=0$ and $a, b$ satisfy the equation $1 / \sqrt{a}+$ $1 / \sqrt{b}=2 / n, n \in N$, (1) becomes

$$
x^{\prime \prime}+a x^{+}-b x^{-}+g(x)=p(t) .
$$

Equation (4) was first introduced by Fučík [8]. Lately, the periodic problem of (4) was widely studied in the literature (see [9-13] and the references cited therein). To deal with the existence of periodic solutions of (4), Dancer [9] introduced a $2 \pi / n$-periodic function

$$
\Phi(\theta)=2 n\left[\frac{g(+\infty)}{a}-\frac{g(-\infty)}{b}\right]-\int_{0}^{2 \pi} p(t) c(t+\theta) d t,
$$

where $c(t)$ is a $2 \pi / n$-periodic function defined by

$$
c(t)= \begin{cases}\frac{1}{\sqrt{a}} \sin (\sqrt{a} t), & 0 \leq t \leq \frac{\pi}{\sqrt{a}}, \\ -\sqrt{\frac{1}{b}} \sin \left[\sqrt{b}\left(t-\frac{\pi}{\sqrt{a}}\right)\right], & \frac{\pi}{\sqrt{a}} \leq t \leq \frac{2 \pi}{n} .\end{cases}
$$

Obviously, $c(t)$ is a periodic solution of the equation $x^{\prime \prime}+$ $a x^{+}-b x^{-}=0$ satisfying the initial value $x(0)=0, x^{\prime}(0)=1$. It was proved in [9] that (4) has at least one $2 \pi$-periodic solution provided that $\Phi$ has a constant sign in $[0,2 \pi / n)$.

In the present paper, we will deal with the periodic solutions of (1) under condition $(g)$. Owing to the appearance 
of the asymmetric nonlinearity $a x^{+}-b x^{-}$, the methods in $[4,5]$ are no longer valid. To overcome this difficulty, we embed (1) into an operator equation with the form $L x=$ $N(x, \lambda)$ instead of $L x=\lambda N x$ as in $[4,5]$. We first prove a continuation lemma and then apply this continuation lemma to prove the existence of periodic solution of (1).

Let us denote

$$
\nu=\tau\left(\bmod \frac{2 \pi}{n}\right)
$$

Obviously, we have

$$
0 \leq v<\frac{2 \pi}{n}
$$

We obtain the following result.

Theorem 1. Assume that condition $(g)$ holds and $0 \leq v \leq$ $\min \{\pi / \sqrt{a}, \pi / \sqrt{b}\}$. Then (1) has at least one $2 \pi$-periodic solution provided that either

$$
\begin{aligned}
& n g(-\infty)\left(\frac{1-\cos \sqrt{a} v}{a}-\frac{1+\cos \sqrt{b} v}{b}\right) \\
& \quad+n g(+\infty)\left(\frac{1+\cos \sqrt{a} v}{a}-\frac{1-\cos \sqrt{b} v}{b}\right) \\
& \neq \int_{0}^{2 \pi} p(t) c(t+\theta) d t, \quad \forall \theta \in[0,2 \pi]
\end{aligned}
$$

or

$$
\begin{gathered}
n(g(-\infty)-g(+\infty))\left(\frac{\sin \sqrt{a} \nu}{\sqrt{a}}+\frac{\sin \sqrt{b} \nu}{\sqrt{b}}\right) \\
\neq \int_{0}^{2 \pi} p(t) s(t+\theta) d t, \quad \forall \theta \in[0,2 \pi]
\end{gathered}
$$

holds, where the function $s$ is defined by $s(t)=c^{\prime}(t), t \in R$.

Remark 2. In the case when $\min \{\pi / \sqrt{a}, \pi / \sqrt{b}\} \leq \nu<2 \pi / n$, we can obtain the similar sufficient conditions. For brevity, we omit the detailed description.

Remark 3. Obviously, if $\nu=0$ or $\tau=2 k \pi / n, k=0,1,2, \ldots, n-$ 1 , then the first inequality of Theorem 1 reduces to the condition as in [8]; namely,

$$
\begin{array}{r}
2 n\left[\frac{g(+\infty)}{a}-\frac{g(-\infty)}{b}\right] \neq \int_{0}^{2 \pi} p(t) c(t+\theta) d t \\
\forall \theta \in[0,2 \pi] .
\end{array}
$$

Throughout this paper, we always use $\mathbf{R}$ to denote the real number set. For a multivariate function $\zeta$ depending on $r$, the notation $\zeta=o(1)$ always means that, for $r \rightarrow \infty, \zeta \rightarrow 0$ holds uniformly with respect to other variables, whereas $\zeta=$ $O(1)$ (or $\zeta=O\left(r^{-1}\right)$ ) always means that $\zeta$ (or $r \cdot \zeta$ ) is bounded for $r$ large enough. For any continuous $2 \pi$-periodic function $\phi(t)$, we always set $\|\phi\|_{\infty}=\max _{0 \leq t \leq 2 \pi}|\phi(t)|$.

\section{Preliminary Lemmas}

We now embed (1) into a family of equations with one parameter $\lambda \in[0,1]$,

$$
x^{\prime \prime}+a x^{+}-b x^{-}+(1-\lambda) \psi\left(x^{\prime}\right)+\lambda g(x(t-\tau))=\lambda p(t),
$$

where $\psi: R \rightarrow R$ is continuous and satisfies the sign condition as follows:

$$
\psi(x) x>0, \quad \forall x \in R, \quad x \neq 0 .
$$

Lemma 4. Suppose that there exist two positive constants $M_{1}$ and $M_{2}$ such that, for any $2 \pi$-periodic solution $x(t)$ of $(12)$, the following conditions hold:

$$
\|x\|_{\infty}<M_{1}, \quad\left\|x^{\prime}\right\|_{\infty}<M_{2} .
$$

Then (1) has at least one $2 \pi$-periodic solution.

Proof. We follow an argument in [14] to prove Lemma 4. At first, we introduce some notations. Let $X$ and $Y$ be two Banach spaces defined by

$$
\begin{gathered}
X=\left\{x \in C^{1}(\mathbf{R}, \mathbf{R}): x(t+2 \pi)=x(t), \forall t \in \mathbf{R}\right\}, \\
Y=\{y \in C(\mathbf{R}, \mathbf{R}): y(t+2 \pi)=y(t), \forall t \in \mathbf{R}\},
\end{gathered}
$$

with the norms

$$
\|x\|_{X}=\max \left\{\|x\|_{\infty},\left\|x^{\prime}\right\|_{\infty}\right\}, \quad\|y\|_{Y}=\|y\|_{\infty} .
$$

Define a linear operator by

$$
L: D(L) \subset X \rightarrow Y, \quad L x=x^{\prime \prime},
$$

where $D(L)=\left\{x \in X: x^{\prime \prime} \in C(\mathbf{R}, \mathbf{R})\right\}$, and a nonlinear operator $N: X \times[0,1] \rightarrow Y$,

$$
\begin{aligned}
N(x, \lambda)(t)= & -\left(a x^{+}-b x^{-}\right)-(1-\lambda) \psi\left(x^{\prime}\right) \\
& -\lambda g(x(t-\tau))+\lambda p(t) .
\end{aligned}
$$

It is easy to see that

$$
\operatorname{Ker} L=\mathbf{R}, \quad \operatorname{Im} L=\left\{y \in Y: \int_{0}^{T} y(t) d t=0\right\} .
$$

It follows that $L$ is a Fredholm mapping of index zero.

Let us define two continuous projectors $P: X \rightarrow \operatorname{Ker} L$ and $Q: Y \rightarrow Y$ by setting

$$
P x=x(0), \quad Q y=\frac{1}{T} \int_{0}^{T} y(t) d t .
$$

Set $L_{P}=\left.L\right|_{D(L) \cap K e r P} \rightarrow \operatorname{Im} L$. Then $L_{P}$ is an algebraic isomorphism, and we define $K_{P}: \operatorname{Im} L \rightarrow D(L)$ by

$$
K_{P}=L_{P}^{-1}
$$


Clearly, we have that, for any $y \in \operatorname{Im} L$,

$$
\left(K_{P} y\right)(t)=-\frac{t}{T} \int_{0}^{T}(t-s) y(s) d s+\int_{0}^{t}(t-s) y(s) d s .
$$

For any open bounded set $\Omega \subset X$, we can prove by standard arguments that $K_{P}(I-Q) N$ and $Q N$ are relatively compact on the closure $\bar{\Omega}$. Therefore, $N$ is $L$-compact on $\bar{\Omega}$.

It is noted that (12), together with the $2 \pi$-periodic boundary condition, is equivalent to the operator equation

$$
L x=N(x, \lambda) .
$$

Let $\Omega \subset X$ be the open bounded set defined by

$$
\Omega=\left\{x \in X:\|x\|_{\infty}<M_{1},\left\|x^{\prime}\right\|_{\infty}<M_{2}\right\} .
$$

From (14), we have

$$
L x \neq N(x, \lambda), \quad \text { for } x \in \partial \Omega \cap D(L), \lambda \in[0,1] .
$$

Since $L$ is a Fredholm operator with index zero and $N$ is $L$ compact on $\bar{\Omega} \times[0,1]$, we get from the homotopic invariance of the coincidence degree that

$$
D_{L}(L-N(\cdot, 1), \Omega)=D_{L}(L-N(\cdot, 0), \Omega) .
$$

Next, we will compute $D_{L}(L-N(\cdot, 0), \Omega)$. To this end, we introduce an auxiliary operator $S: \bar{\Omega} \times[0,1] \rightarrow Y$ defined by

$$
S(x, \mu)=-\left(a x^{+}-b x^{-}\right)-\psi\left(x^{\prime}\right)-\mu x^{\prime} .
$$

Clearly, $S$ is $L$-compact on $\bar{\Omega} \times[0,1]$ and

$$
S(x, 0)=N(x, 0), \quad \text { for } x \in \bar{\Omega} .
$$

Now, we will prove that

$$
L x \neq S(x, \mu), \quad \text { for } x \in \partial \Omega \cap \operatorname{dom} L, \mu \in[0,1] .
$$

Obviously, it follows from (25) and (28) that

$$
L x \neq S(x, 0), \quad \text { for } x \in \partial \Omega .
$$

On the other hand, if $x \in \operatorname{dom} L$ is a solution of $L x=S(x, \mu)$, then $x$ satisfies the equation as follows:

$$
x^{\prime \prime}+\mu x^{\prime}+\left(a x^{+}-b x^{-}\right)+\psi\left(x^{\prime}\right)=0 .
$$

Multiplying both sides of (31) by $x^{\prime}$ and integrating over $[0,2 \pi]$, we get

$$
\mu \int_{0}^{2 \pi} x^{\prime 2}(t) d t+\int_{0}^{2 \pi} \psi\left(x^{\prime}\right) x^{\prime} d t=0 .
$$

If $\mu>0$, then we infer from (13) and (32) that $x^{\prime}(t) \equiv 0$ for every $t \in[0,2 \pi]$. Furthermore, $x(t) \equiv c$ for every $t \in[0,2 \pi]$, where $c$ is a constant. Consequently, we have $x(t) \equiv 0$, and then $x \in \Omega$.
From the homotopic invariance of the coincidence degree, we have

$$
D_{L}(L-S(\cdot, 0), \Omega)=D_{L}(L-S(\cdot, 1), \Omega)
$$

In the following, we will compute $D_{L}(L-S(\cdot, 1), \Omega)$. To this end, we use the equality [15] as follows:

$$
\left|D_{L}(L-S(\cdot, 1), \Omega)\right|=\left|d_{B}\left(-\left.Q S(\cdot, 1)\right|_{\operatorname{Ker} L}, \Omega \cap \operatorname{Ker} L, 0\right)\right|,
$$

which holds provided that the following conditions are satisfied,

$$
\begin{gathered}
L x \neq \lambda S(x, 1), \quad \forall x \in \partial \Omega \cap \operatorname{dom} L, \lambda \in(0,1], \\
Q S(x, 1) \neq 0, \quad \forall x \in \partial \Omega \cap \operatorname{Ker} L .
\end{gathered}
$$

In what follows, we will prove that conditions (35) and (36) are satisfied. In fact, if $x \in \partial \Omega \cap \operatorname{dom} L$ is a solution of $L x=$ $\lambda S(x, 1)$, then $x(t)$ satisfies the equation as follows:

$$
x^{\prime \prime}(t)+\lambda x^{\prime}+\lambda\left(a x^{+}-b x^{-}\right)+\lambda \psi\left(x^{\prime}\right)=0
$$

Using the same method as before, we can get $x \in \Omega$. This is a contradiction. To check condition (36), we notice that if $x \in \partial \Omega \cap \operatorname{Ker} L$, then $x(t)=c^{\prime}$ with $\left|c^{\prime}\right|=M_{1}$. Hence, we have that, for $x \in \partial \Omega \cap \operatorname{Ker} L$,

$$
Q S(x, 1)=\frac{1}{T} \int_{0}^{T}\left(-a x^{+}+b x^{-}\right) d t=-a c^{\prime} \quad \text { or } \quad-b c^{\prime} \neq 0 \text {. }
$$

Finally, we can easily calculate the Brouwer degree $d_{B}\left(-\left.Q S(\cdot, 1)\right|_{\operatorname{Ker} L}, \Omega \cap \operatorname{Ker} L, 0\right)$ and obtain

$$
d_{B}\left(-\left.Q S(\cdot, 1)\right|_{\operatorname{Ker} L}, \Omega \cap \operatorname{Ker} L, 0\right)=1 .
$$

Therefore, we have

$$
D_{L}(L-N(\cdot, 1), \Omega) \neq 0 \text {. }
$$

Consequently, the equation

$$
L x=N(x, 1)
$$

has at least one $2 \pi$-periodic solution. Equivalently, (1) has at least one $2 \pi$-periodic solution.

Remark 5. In (12), if $\psi$ satisfies the following condition,

$$
x \psi(x)<0, \quad \forall x \in \mathbf{R}, x \neq 0,
$$

then the conclusion of Lemma 4 still holds. This claim can be proved by using the same method as the one used for proving Lemma 4 . In fact, we only need to modify the term $-\mu x^{\prime}$ in the auxiliary operator $S(x, \mu)$ to the term $\mu x^{\prime}$. 


\section{Periodic Solutions of Duffing Equation with a Deviating Argument}

At first, we choose a continuous function $\psi: R \rightarrow R$ satisfying

$$
\lim _{x \rightarrow \pm \infty} \psi(x)=\psi( \pm \infty)
$$

where $\psi( \pm \infty) \in R$ are constants. Moreover, $\psi$ satisfies condition (13).

Considering the equivalent system of (12),

$$
\begin{gathered}
x^{\prime}=y, \\
y^{\prime}=-\left(a x^{+}-b x^{-}\right)-\lambda g(x(t-\tau))-(1-\lambda) \psi\left(x^{\prime}\right) \\
+\lambda p(t) .
\end{gathered}
$$

Let $x(t)$ be any (possible) $2 \pi$-periodic solution of (12). Write $y(t)=x^{\prime}(t)$. Then, $(x(t), y(t))$ is a $2 \pi$-periodic solution of system (44).

In what follows, we will introduce a transformation. To this end, let us denote by $c(t)$ a solution of equation $x^{\prime \prime}+a x^{+}-$ $b x^{-}=0$ satisfying the initial condition $c(0)=0, c^{\prime}(0)=1$. Obviously, $c(t)$ is $2 \pi / n$-periodic. The derivative of $c(t)$ will be denoted by $s(t)=c^{\prime}(t)$. It is easy to check that the following properties are satisfied:

(1) $c(t+2 \pi / n)=c(t), s(t+2 \pi / n)=s(t)$.

(2) $c^{\prime}(t)=s(t), s^{\prime}(t)=-\left(a c^{+}(t)-b c^{-}(t)\right)$.

(3) $s(t)^{2}+a c^{+}(t)^{2}+b c^{-}(t)^{2}=1, \forall t \in R$.

Let us define a mapping $\Phi:(\theta, \rho) \in S^{1} \times(0,+\infty) \rightarrow(x, y) \in$ $R^{2} \backslash\{0\}$ as follows:

$$
x=\rho^{1 / 2} c\left(\frac{\theta}{n}\right), \quad y=\rho^{1 / 2} s\left(\frac{\theta}{n}\right),
$$

where $S^{1}=R / 2 \pi Z$.

Under the transformation $\Phi$, if $|x(t)|+|y(t)| \neq 0, \forall t \in$ $[0,2 \pi]$, then the $2 \pi$-periodic solution $(x(t), y(t))$ of system (44) can be expressed in the form $(\rho(t), \theta(t))$ satisfying the equations as follows:

$$
\begin{aligned}
& \frac{d \rho}{d t} \\
& =-2 \lambda \rho^{1 / 2} \\
& \quad \times\left(g\left(\rho^{1 / 2}(t-\tau) c\left(\frac{\theta(t-\tau)}{n}\right)\right) s\left(\frac{\theta}{n}\right)-p(t) s\left(\frac{\theta}{n}\right)\right) \\
& \quad-2(1-\lambda) \rho^{1 / 2} \psi\left(\rho^{1 / 2} s\left(\frac{\theta}{n}\right)\right) s\left(\frac{\theta}{n}\right),
\end{aligned}
$$

$\frac{d \theta}{d t}$

$$
\begin{aligned}
= & n+n \lambda \rho^{-1 / 2} \\
& \times\left(g\left(\rho^{1 / 2}(t-\tau) c\left(\frac{\theta(t-\tau)}{n}\right)\right) c\left(\frac{\theta}{n}\right)-p(t) c\left(\frac{\theta}{n}\right)\right) \\
& +n(1-\lambda) \rho^{-1 / 2} \psi\left(\rho^{1 / 2} s\left(\frac{\theta}{n}\right)\right) c\left(\frac{\theta}{n}\right) .
\end{aligned}
$$

Let us denote $\left(\rho_{0}, \theta_{0}\right)=(\rho(0), \theta(0))$. From now on, we always assume that $g$ is bounded. From the first equation of (46) we get that

$$
\begin{aligned}
\frac{d \rho^{1 / 2}}{d t}= & -\lambda g\left(\rho^{1 / 2}(t-\tau) c\left(\frac{\theta(t-\tau)}{n}\right)\right) s\left(\frac{\theta}{n}\right) \\
& +\lambda p(t) s\left(\frac{\theta}{n}\right)-(1-\lambda) \psi\left(\rho^{1 / 2} s\left(\frac{\theta}{n}\right)\right) s\left(\frac{\theta}{n}\right) .
\end{aligned}
$$

Therefore, we have

$$
\rho(t)^{1 / 2}=\rho_{0}^{1 / 2}+O(1) .
$$

Furthermore, we get

$$
\rho(t)^{-1 / 2}=\rho_{0}^{-1 / 2}+O\left(\rho_{0}^{-1}\right) .
$$

From the second equation of (46), we have

$$
\frac{d \theta}{d t}=n+O\left(\rho_{0}^{-1 / 2}\right) \text {. }
$$

As a result,

$$
\theta(t)=\theta_{0}+n t+O\left(\rho_{0}^{-1 / 2}\right) .
$$

Substituting (51) in (47), we obtain that, for $t \in[0,2 \pi]$,

$$
\begin{aligned}
\frac{d \rho^{1 / 2}}{d t}= & -\lambda g\left(\rho_{0}^{1 / 2} c\left(t-\tau+\frac{\theta_{0}}{n}\right)+O(1)\right) s\left(t+\frac{\theta_{0}}{n}\right) \\
& +\lambda p(t) s\left(t+\frac{\theta_{0}}{n}\right)-(1-\lambda) \psi\left(\rho^{1 / 2} s\left(\frac{\theta}{n}\right)\right) s\left(\frac{\theta}{n}\right) \\
& +O\left(\rho_{0}^{-1 / 2}\right) .
\end{aligned}
$$

Consequently,

$\rho^{1 / 2}(2 \pi)$

$$
\begin{aligned}
= & \rho_{0}^{1 / 2}-\lambda \int_{0}^{2 \pi} g\left(\rho_{0}^{1 / 2} c\left(t-\tau+\frac{\theta_{0}}{n}\right)+O(1)\right) s\left(t+\frac{\theta_{0}}{n}\right) d t \\
& -(1-\lambda) \int_{0}^{2 \pi} \psi\left(\rho_{0}^{1 / 2} s\left(t+\frac{\theta_{0}}{n}\right)+O(1)\right) s\left(t+\frac{\theta_{0}}{n}\right) d t \\
& +\lambda \int_{0}^{2 \pi} p(t) s\left(t+\frac{\theta_{0}}{n}\right) d t+O\left(\rho_{0}^{-1 / 2}\right) .
\end{aligned}
$$


Similarly, substituting (51) in the second equality of (44), we get that, for $t \in[0,2 \pi]$,

$$
\begin{aligned}
\frac{d \theta}{d t}= & +n \lambda \rho_{0}^{-1 / 2} g\left(\rho_{0}^{1 / 2} c\left(t-\tau+\frac{\theta_{0}}{n}\right)+O(1)\right) \\
& \times c\left(t+\frac{\theta_{0}}{n}\right)-n \lambda \rho_{0}^{-1 / 2} p(t) c\left(t+\frac{\theta_{0}}{n}\right) \\
& +n(1-\lambda) \rho_{0}^{-1 / 2} \psi\left(\rho_{0}^{1 / 2} s\left(t+\frac{\theta_{0}}{n}\right)+O(1)\right) \\
& \times c\left(t+\frac{\theta_{0}}{n}\right)+O\left(\rho_{0}^{-1}\right) .
\end{aligned}
$$

Therefore, we have

$$
\begin{aligned}
& \theta(2 \pi) \\
& =\theta_{0}+2 n \pi+n \lambda \rho_{0}^{-1 / 2} \\
& \quad \times \int_{0}^{2 \pi} g\left(\rho_{0}^{1 / 2} c\left(t-\tau+\frac{\theta_{0}}{n}\right)+O(1)\right) c\left(t+\frac{\theta_{0}}{n}\right) d t \\
& \quad+n(1-\lambda) \rho_{0}^{-1 / 2} \\
& \quad \times \int_{0}^{2 \pi} \psi\left(\rho_{0}^{1 / 2} s\left(t+\frac{\theta_{0}}{n}\right)+O(1)\right) c\left(t+\frac{\theta_{0}}{n}\right) d t \\
& \quad-n \lambda \rho_{0}^{-1 / 2} \int_{0}^{2 \pi} p(t) c\left(t+\frac{\theta_{0}}{n}\right) d t+O\left(\rho_{0}^{-1}\right) .
\end{aligned}
$$

Write

$$
\begin{aligned}
& \psi_{1}\left(\theta_{0}\right)=\int_{0}^{2 \pi} g\left(\rho_{0}^{1 / 2} c\left(t-\tau+\frac{\theta_{0}}{n}\right)+O(1)\right) s\left(t+\frac{\theta_{0}}{n}\right) d t \\
& \psi_{2}\left(\theta_{0}\right)=\int_{0}^{2 \pi} g\left(\rho_{0}^{1 / 2} c\left(t-\tau+\frac{\theta_{0}}{n}\right)+O(1)\right) c\left(t+\frac{\theta_{0}}{n}\right) d t \\
& \psi_{3}\left(\theta_{0}\right)=\int_{0}^{2 \pi} \psi\left(\rho_{0}^{1 / 2} s\left(t+\frac{\theta_{0}}{n}\right)+O(1)\right) s\left(t+\frac{\theta_{0}}{n}\right) d t \\
& \psi_{4}\left(\theta_{0}\right)=\int_{0}^{2 \pi} \psi\left(\rho_{0}^{1 / 2} s\left(t+\frac{\theta_{0}}{n}\right)+O(1)\right) c\left(t+\frac{\theta_{0}}{n}\right) d t
\end{aligned}
$$

Recalling that $\nu=\tau(\bmod (2 \pi / n))$ and $0 \leq \nu<2 \pi / n$, we have the following estimates.
Lemma 6. Assume that condition $(g)$ holds. Then, for $\rho_{0} \rightarrow$ $+\infty$,

$$
\begin{aligned}
& \psi_{1}\left(\theta_{0}\right)
\end{aligned}
$$

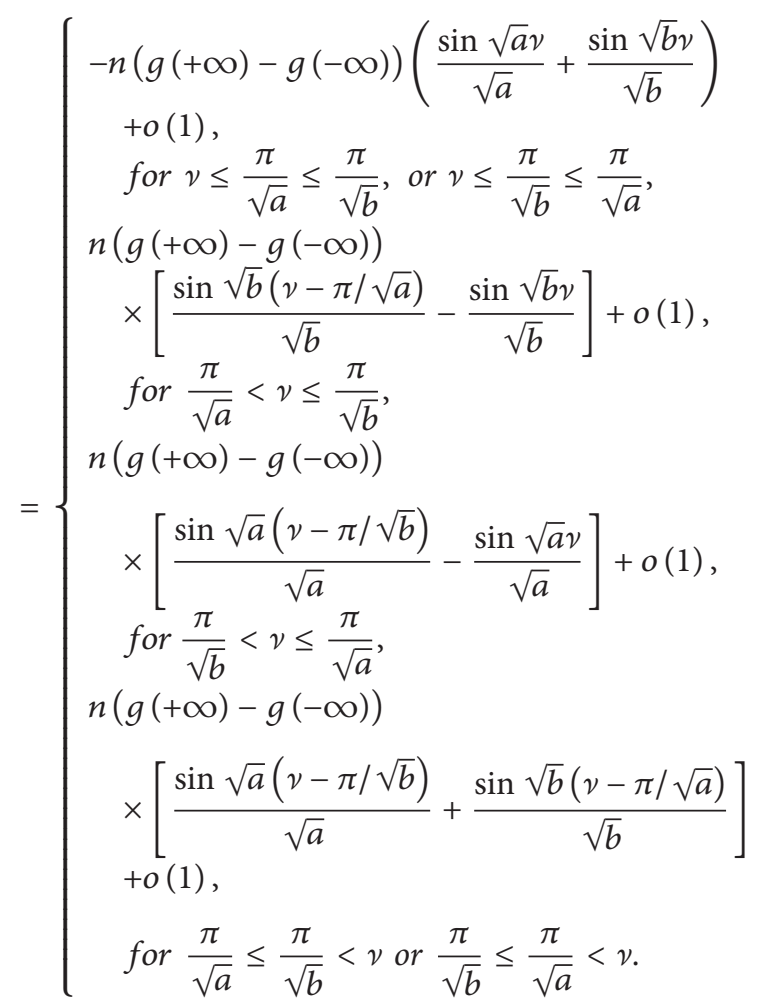

Proof. We only give the proof for the case $0 \leq \nu \leq \pi / \sqrt{a} \leq$ $\pi / \sqrt{b}<2 \pi / n$. The other cases can be treated similarly. Since $s(t)$ is $2 \pi / n$-periodic, it follows from the expression of $\psi_{1}\left(\theta_{0}\right)$ that

$$
\begin{aligned}
\psi_{1}\left(\theta_{0}\right) & =\int_{0}^{2 \pi} g\left(\rho_{0}^{1 / 2} c\left(t-\tau+\frac{\theta_{0}}{n}\right)+O(1)\right) s\left(t+\frac{\theta_{0}}{n}\right) d t \\
& =\int_{0}^{2 \pi} g\left(\rho_{0}^{1 / 2} c(u)+O(1)\right) s(u+\tau) d u \\
& =\int_{0}^{2 \pi} g\left(\rho_{0}^{1 / 2} c(u)+O(1)\right) s(u+v) d u
\end{aligned}
$$

From the dominated convergent theorem, we have that, for $\rho_{0} \rightarrow \infty$,

$$
\begin{aligned}
& \psi_{1}\left(\theta_{0}\right) \\
& =n g(+\infty) \int_{0}^{\pi / \sqrt{a}} s(u+v) d u+n g(-\infty) \\
& \quad \times \int_{\pi / \sqrt{a}}^{2 \pi / n} s(u+v) d u+o(1) \\
& =n g(+\infty)\left[\int_{0}^{\pi / \sqrt{a}-v} s(u+v) d u+\int_{\pi / \sqrt{a}-v}^{\pi / \sqrt{a}} s(u+v) d u\right]
\end{aligned}
$$




$$
\begin{aligned}
& +n g(-\infty)\left[\int_{\pi / \sqrt{a}}^{2 \pi / n-v} s(u+v) d u\right. \\
& \left.+\int_{2 \pi / n-v}^{2 \pi / n} s(u+v) d u\right]+o(1) \\
& =-n g(+\infty)\left(\frac{\sin \sqrt{a} v}{\sqrt{a}}+\frac{\sin \sqrt{b} v}{\sqrt{b}}\right) \\
& +n g(-\infty)\left(\frac{\sin \sqrt{a} v}{\sqrt{a}}+\frac{\sin \sqrt{b} v}{\sqrt{b}}\right)+o(1) \\
& =-n(g(+\infty)-g(-\infty))\left(\frac{\sin \sqrt{a} v}{\sqrt{a}}+\frac{\sin \sqrt{b} v}{\sqrt{b}}\right)+o(1)
\end{aligned}
$$

Lemma 7. Assume that condition $(g)$ holds. Then, for $\rho_{0} \rightarrow$ $+\infty$,

$\psi_{2}\left(\theta_{0}\right)$

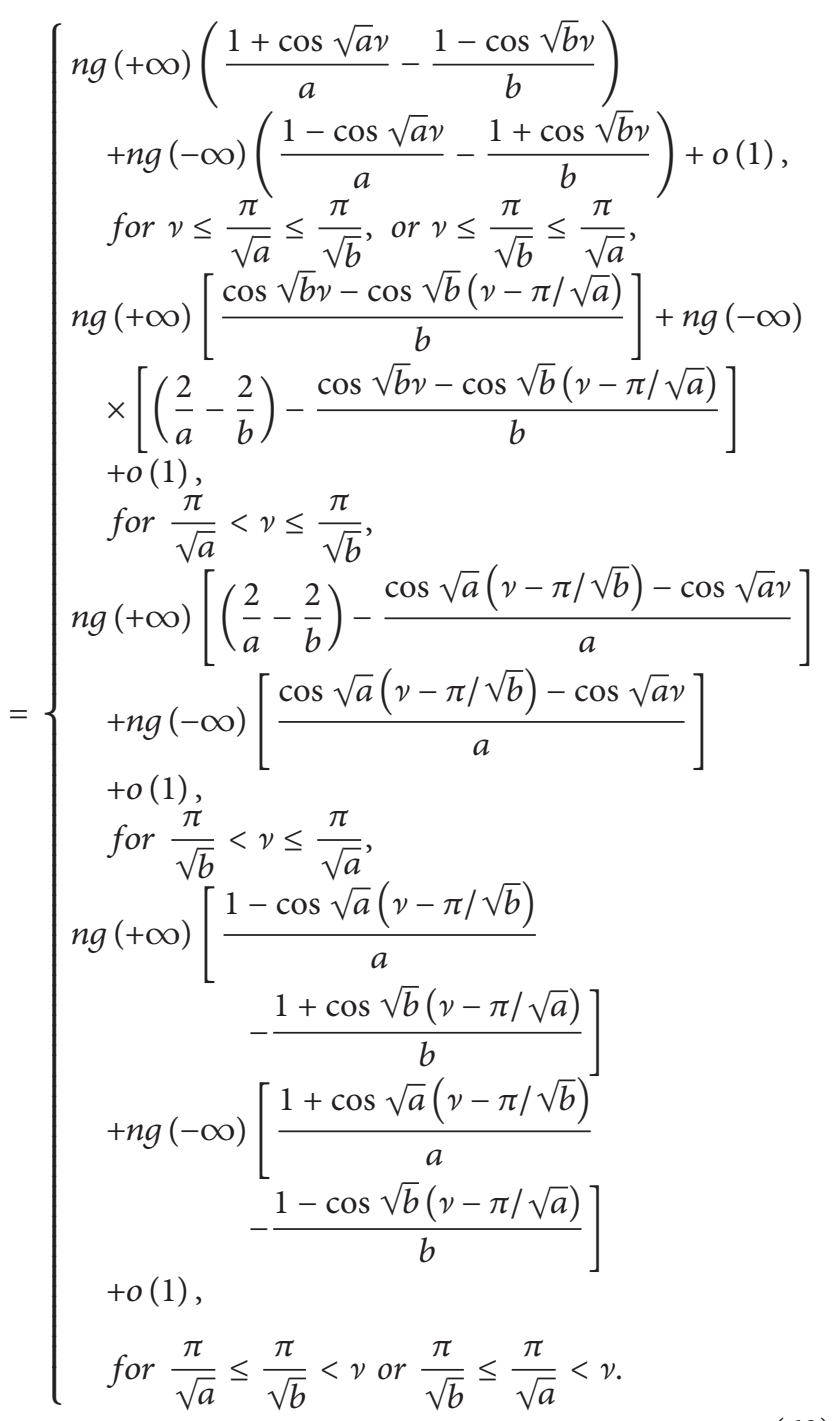

Proof. We also only give the proof for the case $0 \leq$ $v \leq \pi / \sqrt{a} \leq \pi / \sqrt{b}<2 \pi / n$. The other cases can be treated similarly. Since $c(t)$ is $2 \pi / n$-periodic, it follows from the expression of $\psi_{2}\left(\theta_{0}\right)$ and the dominated convergent theorem that, for $\rho_{0} \rightarrow \infty$,

$$
\begin{aligned}
\psi_{2}( & \left.\theta_{0}\right) \\
= & \int_{0}^{2 \pi} g\left(\rho_{0}^{1 / 2} c\left(t-\tau+\frac{\theta_{0}}{n}\right)+O(1)\right) c\left(t+\frac{\theta_{0}}{n}\right) d t \\
= & \int_{0}^{2 \pi} g\left(\rho_{0}^{1 / 2} c(u)+O(1)\right) c(u+\tau) d u \\
= & \int_{0}^{2 \pi} g\left(\rho_{0}^{1 / 2} c(u)+O(1)\right) c(u+v) d u \\
= & n g(+\infty) \int_{0}^{\pi / \sqrt{a}} c(u+v) d u \\
& +n g(-\infty) \int_{\pi / \sqrt{a}}^{2 \pi / n}(u+v) d u+o(1) \\
= & n g(+\infty) \\
& +n g(-\infty)\left(\frac{1-\cos \sqrt{a} v}{a}-\frac{1+\cos \sqrt{b} v}{b}\right)+o(1) \\
& \times\left[\int_{0}^{\pi / \sqrt{a}-v} c(u+v) d u+\int_{\pi / \sqrt{a}-v}^{\pi / \sqrt{a}} c(u+v) d u\right] \\
& +n g(-\infty) \\
& \times\left[\int_{\pi / \sqrt{a}}^{2 \pi / n-v} c(u+v) d u+\int_{2 \pi / n-v}^{2 \pi / n} c(1)\right. \\
& n g(+\infty)\left(\frac{1+\cos \sqrt{a} v}{a}-\frac{1-\cos \sqrt{b} v}{b}\right) \\
& \\
& \\
& \\
&
\end{aligned}
$$

Lemma 8. Assume that condition (43) holds. Then, for $\rho_{0} \rightarrow$ $+\infty$,

$$
\begin{aligned}
& \psi_{3}\left(\theta_{0}\right)=2[\psi(+\infty)-\psi(-\infty)]+o(1) \\
& \psi_{4}\left(\theta_{0}\right)=n[\psi(+\infty)+\psi(-\infty)]\left(\frac{1}{a}-\frac{1}{b}\right)+o(1)
\end{aligned}
$$

Proof. From the expression of $\psi_{3}\left(\theta_{0}\right)$ and the dominated convergent theorem we have that, for $\rho_{0} \rightarrow \infty$,

$$
\begin{aligned}
\psi_{3} & \left(\theta_{0}\right) \\
= & \int_{0}^{2 \pi} \psi\left(\rho_{0}^{1 / 2} s\left(t+\frac{\theta_{0}}{n}\right)+O(1)\right) s\left(t+\frac{\theta_{0}}{n}\right) d t \\
= & \int_{0}^{2 \pi} \psi\left(\rho_{0}^{1 / 2} s(u)+O(1)\right) s(u) d u
\end{aligned}
$$




$$
\begin{aligned}
= & n \psi(+\infty)\left(\int_{0}^{\pi / 2 \sqrt{a}} s(u) d u+\int_{\pi / \sqrt{a}+\pi / 2 \sqrt{b}}^{2 \pi / n} s(u) d u\right) \\
& +n \psi(-\infty)\left(\int_{\pi / 2 \sqrt{a}}^{\pi / \sqrt{a}} s(u) d u+\int_{\pi / \sqrt{a}}^{\pi / \sqrt{a}+\pi / 2 \sqrt{b}} s(u) d u\right) \\
& +o(1) \\
= & 2[\psi(+\infty)-\psi(-\infty)]+o(1) .
\end{aligned}
$$

Similarly, we have that, for $\rho_{0} \rightarrow+\infty$,

$$
\begin{aligned}
\psi_{4}\left(\theta_{0}\right) & \int_{0}^{2 \pi} \psi\left(\rho_{0}^{1 / 2} s\left(t+\frac{\theta_{0}}{n}\right)+O(1)\right) c\left(t+\frac{\theta_{0}}{n}\right) d t \\
= & \int_{0}^{2 \pi} \psi\left(\rho_{0}^{1 / 2} s(u)+O(1)\right) c(u) d u \\
= & n \psi(+\infty)\left(\int_{0}^{\pi / 2 \sqrt{a}} c(u) d u+\int_{(\pi / \sqrt{a})+(\pi / 2 \sqrt{b})}^{2 \pi / n} c(u) d u\right) \\
& +n \psi(-\infty)\left(\int_{\pi / 2 \sqrt{a}}^{\pi / \sqrt{a}} c(u) d u+\int_{\pi / \sqrt{a}}^{(\pi / \sqrt{a})+(\pi / 2 \sqrt{b})} c(u) d u\right) \\
& +o(1)=n[\psi(+\infty)+\psi(-\infty)]\left(\frac{1}{a}-\frac{1}{b}\right)+o(1) .
\end{aligned}
$$

Proof of Theorem 1. We proceed to prove Theorem 1 in two different cases.

(1) Assume that the first inequality of Theorem 1 holds. Without loss of generality, we assume

$$
\begin{aligned}
& n g(-\infty)\left(\frac{1-\cos \sqrt{a} v}{a}-\frac{1+\cos \sqrt{b} v}{b}\right) \\
& \quad+n g(+\infty)\left(\frac{1+\cos \sqrt{a} v}{a}-\frac{1-\cos \sqrt{b} v}{b}\right) \\
& >\int_{0}^{2 \pi} p(t) c(t+\theta) d t, \quad \forall \theta \in[0,2 \pi] .
\end{aligned}
$$

Let us set

$$
\begin{aligned}
\eta(\theta)= & n g(-\infty)\left(\frac{1-\cos \sqrt{a} \nu}{a}-\frac{1+\cos \sqrt{b} \nu}{b}\right) \\
& +n g(+\infty)\left(\frac{1+\cos \sqrt{a} \nu}{a}-\frac{1-\cos \sqrt{b} \nu}{b}\right) \\
& -\int_{0}^{2 \pi} p(t) c(t+\theta) d t>0, \quad \theta \in[0,2 \pi] .
\end{aligned}
$$

We now choose a function $\psi$ satisfying (43) and (13). Moreover, $\psi( \pm \infty)$ satisfy

$$
\mu=[\psi(+\infty)+\psi(-\infty)]\left(\frac{1}{a}-\frac{1}{b}\right)>0 .
$$

Then we infer from Lemmas 7 and 8 that, for $\rho_{0} \rightarrow \infty$,

$$
\begin{aligned}
\theta(2 \pi)= & \theta_{0}+2 n \pi+n \rho_{0}^{-1 / 2}\left[\lambda \eta\left(\frac{\theta_{0}}{n}\right)+n(1-\lambda) \mu\right] \\
& +o\left(\rho_{0}^{-1 / 2}\right) .
\end{aligned}
$$

Since $\eta(\theta)>0, \theta \in[0,2 \pi]$, and $\mu>0$, there exists a constant $\gamma>0$ such that, for $\theta \in[0,2 \pi]$ and $\lambda \in[0,1]$,

$$
\lambda \eta(\theta)+n(1-\lambda) \mu \geq \gamma .
$$

From (68) and (69) we have that, for $\rho_{0} \rightarrow \infty$,

$$
\theta(2 \pi)=\theta_{0}+2 n \pi+o(1), \quad \theta(2 \pi)>\theta_{0}+2 n \pi .
$$

Consequently, there exists a constant $M>0$ such that if $(\rho(t), \theta(t))$ is a $2 \pi$-periodic solution of system (46), then $\rho(t) \leq M, t \in[0,2 \pi]$. Furthermore, there exist constants $M_{1}>0$ and $M_{2}>0$ such that if $x(t)$ is a $2 \pi$-periodic solution of (12), then

$$
\|x\|_{\infty}<M_{1}, \quad\left\|x^{\prime}\right\|_{\infty}<M_{2}
$$

From Lemma 4 , we know that (1) has at least one $2 \pi$-periodic solution.

(2) We assume that the second inequality of Theorem 1 holds. Without loss of generality, we assume

$$
\begin{gathered}
n(g(-\infty)-g(+\infty))\left(\frac{\sin \sqrt{a} \nu}{\sqrt{a}}+\frac{\sin \sqrt{b} v}{\sqrt{b}}\right) \\
>\int_{0}^{2 \pi} p(t) s(t+\theta) d t, \quad \forall \theta \in[0,2 \pi] .
\end{gathered}
$$

Let us set

$$
\begin{aligned}
\zeta(\theta)= & n(g(-\infty)-g(+\infty))\left(\frac{\sin \sqrt{a} \nu}{\sqrt{a}}+\frac{\sin \sqrt{b} v}{\sqrt{b}}\right) \\
& -\int_{0}^{2 \pi} p(t) s(t+\theta) d t>0, \quad \theta \in[0,2 \pi] .
\end{aligned}
$$

Similarly, we choose a continuous function $\psi$ satisfying (43) and (13). Moreover, $\psi( \pm \infty)$ satisfy

$$
\mu^{\prime}=\psi(+\infty)-\psi(-\infty)>0
$$

Then we infer from Lemmas 7 and 8 that, for $\rho_{0} \rightarrow \infty$,

$$
\rho^{1 / 2}(2 \pi)=\rho_{0}^{1 / 2}-\left[\lambda \zeta\left(\frac{\theta_{0}}{n}\right)+2(1-\lambda) \mu^{\prime}\right]+o(1) .
$$

Since $\zeta(\theta)>0, \theta \in[0,2 \pi]$, and $\mu^{\prime}>0$, there exists a constant $\gamma^{\prime}>0$ such that, for $\theta \in[0,2 \pi]$ and $\lambda \in[0,1]$ and $\rho_{0} \rightarrow \infty$,

$$
\lambda \zeta(\theta)+2(1-\lambda) \mu^{\prime} \geq \gamma^{\prime}
$$

From (75) and (76) we have that, for sufficiently large $\rho_{0}$,

$$
\rho^{1 / 2}(2 \pi) \leq \rho_{0}^{1 / 2}-\frac{\gamma^{\prime}}{2} .
$$


Consequently, there exists a constant $M^{\prime}>0$ such that if $(\rho(t), \theta(t))$ is a $2 \pi$-periodic solution of system (46), then $\rho(t) \leq M^{\prime}, t \in[0,2 \pi]$. Furthermore, there exist constants $M_{1}^{\prime}>0$ and $M_{2}^{\prime}>0$ such that if $x(t)$ is a $2 \pi$ periodic solution of (12), then

$$
\|x\|_{\infty}<M_{1}^{\prime}, \quad\left\|x^{\prime}\right\|_{\infty}<M_{2}^{\prime} .
$$

From Lemma 4 we know that (1) has at least one $2 \pi$ periodic solution.

\section{Acknowledgments}

This research is supported by Research Fund for the Doctoral Program of Higher Education of China, no. 11AA0013, Beijing Natural Science Foundation (Existence and multiplicity of periodic solutions in nonlinear oscillations), no. 1112006, and the Grant of Beijing Education Committee Key Project, no. KZ201310028031.

\section{References}

[1] X. Huang and Z. Xiang, "On the existence of $2 \pi$ periodic solutions of Duffing type equation $x^{\prime \prime}(t)=g(x(t-\tau))=p(t)$," Chinese Science Bulletin, vol. 39, pp. 201-203, 1994 (Chinese).

[2] S. Ma, Z. Wang, and J. Yu, "An abstract existence theorem at resonance and its applications," Journal of Differential Equations, vol. 145, no. 2, pp. 274-294, 1998.

[3] R. K. Nagle and M. E. Parrott, "Bounded perturbations with multiple delays of forced harmonic oscillators at resonance," Differential and Integral Equations, vol. 5, no. 6, pp. 1407-1418, 1992.

[4] W. G. Ge, "On the existence of harmonic solutions of Liénard systems," Nonlinear Analysis. Theory, Methods \& Applications A, vol. 16, no. 2, pp. 183-190, 1991.

[5] B. Liu and L. Huang, "Existence and uniqueness of periodic solutions for a kind of Liénard equation with a deviating argument," Applied Mathematics Letters, vol. 21, no. 1, pp. 5662, 2008.

[6] T. A. Burton and B. Zhang, "Boundedness, periodicity, and convergence of solutions in a retarded Liénard equation," Annali di Matematica Pura ed Applicata, vol. 165, pp. 351-368, 1993.

[7] A. C. Lazer and D. E. Leach, "Bounded perturbations of forced harmonic oscillators at resonance," Annali di Matematica Pura ed Applicata, vol. 82, pp. 49-68, 1969.

[8] S. Fučík, Solvability of Nonlinear Equations and Boundary Value Problems, vol. 4, Reidel, Dordrecht, The Netherlands, 1980.

[9] E. N. Dancer, "Boundary-value problems for weakly nonlinear ordinary differential equations," Bulletin of the Australian Mathematical Society, vol. 15, no. 3, pp. 321-328, 1976.

[10] C. Fabry and A. Fonda, "Nonlinear resonance in asymmetric oscillators," Journal of Differential Equations, vol. 147, no. 1, pp. 58-78, 1998.

[11] A. Capietto and Z. Wang, "Periodic solutions of Liénard equations with asymmetric nonlinearities at resonance," Journal of the London Mathematical Society, vol. 68, no. 1, pp. 119-132, 2003.

[12] Z. Wang, "Periodic solutions of the second order differential equations with asymmetric nonlinearities depending on the derivatives," Discrete and Continuous Dynamical Systems, vol. 9, no. 3, pp. 751-770, 2003.
[13] A. C. Lazer and P. J. McKenna, "Existence, uniqueness, and stability of oscillations in differential equations with asymmetric nonlinearities," Transactions of the American Mathematical Society, vol. 315, no. 2, pp. 721-739, 1989.

[14] T. R. Ding, R. Iannacci, and F. Zanolin, "On periodic solutions of sublinear Duffing equations," Journal of Mathematical Analysis and Applications, vol. 158, no. 2, pp. 316-332, 1991.

[15] R. E. Gaines and J. L. Mawhin, Coincidence Degree, and Nonlinear Differential Equations, Springer, Berlin, Germany, 1977. 


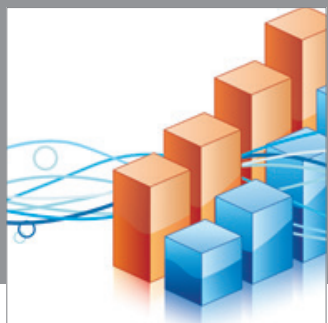

Advances in

Operations Research

mansans

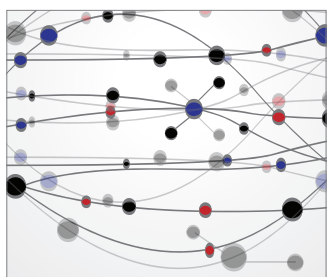

The Scientific World Journal
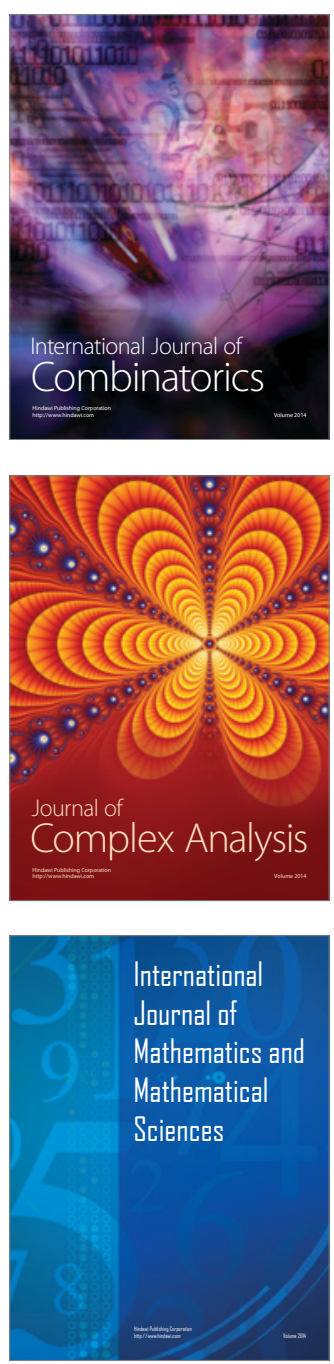
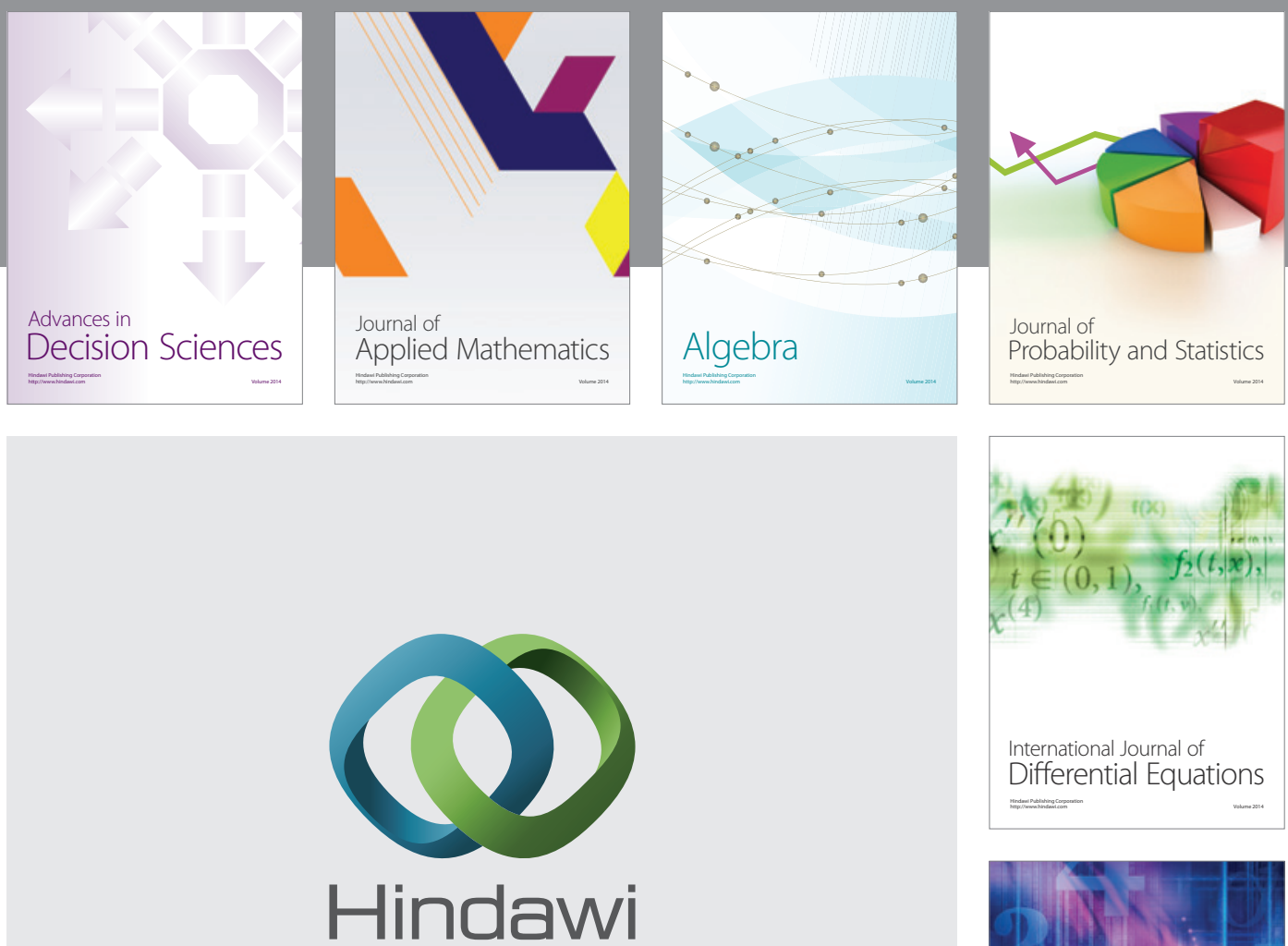

Submit your manuscripts at http://www.hindawi.com
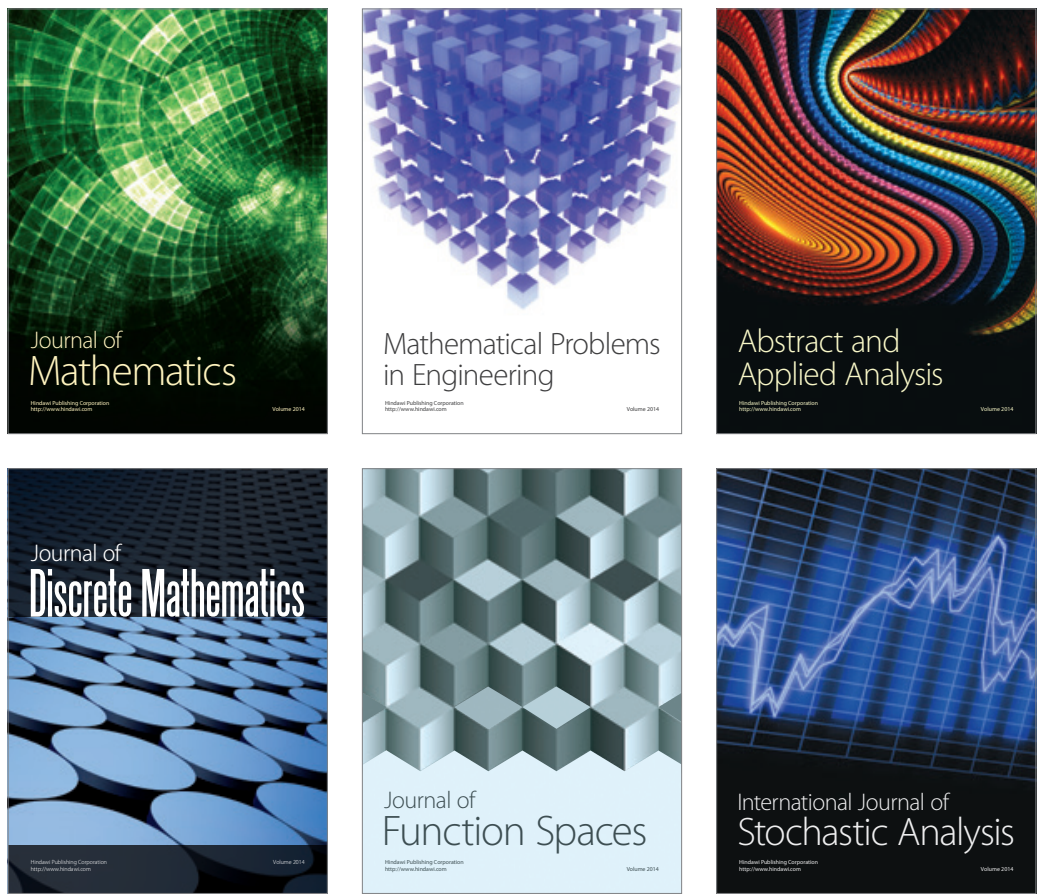

Journal of

Function Spaces

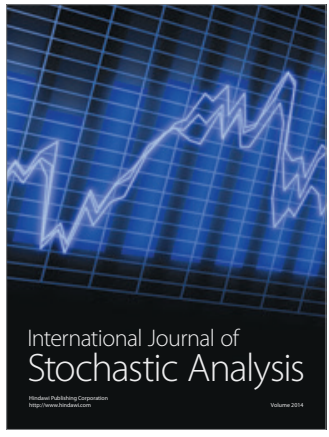

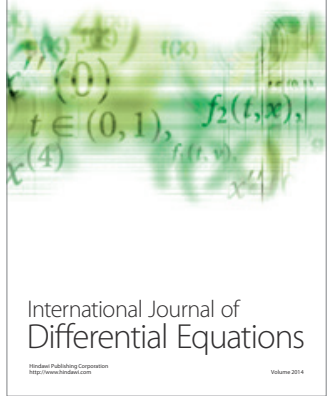
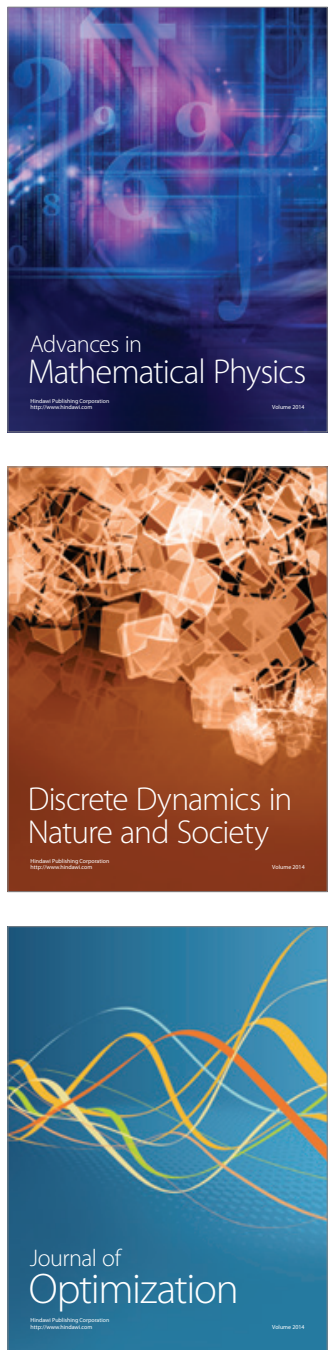